\title{
Simple Microwave Method for Detecting Water Holdup
}

\author{
Sheikh S. I., Alqurashi K. Y., Ragheb H.A. and Babelli I.
}

A microwave sensor is designed to measure the resonance behavior of the hydrocarbon mixture to determine the water holdup of a near horizontal oil carrying pipeline. This technique is particularly useful for detecting very small water holdup $(<5 \%)$ as demonstrated by simulated and experimental results.

Index Terms: Microwave, Reflection, Multiphase flow, Water Holdup, Oil pipeline

Introduction: Oil production generally results in multiphase flow of oil, water and gas. The complex dielectric properties of this mixture are normally analyzed to find the phase fractions, as a basis for determining flow rates and concentrations [1]. Popular electrical methods in determining the dielectric properties of the mixture include inductance, resistance and capacitance measurements, which are often incapable of providing accurate concentration estimates, particularly in very low or very high water cut production wells [2]. Radio frequency (RF) transmission measurements are also used to detect sand, water and gas levels within multiphase flow in pipelines [3]. In this paper, a simple microwave technique is used to excite various combination of two-phase liquid and observe their complex electrical properties. Since electromagnetic scattering is governed by the geometrical and electrical properties of the pipe and its contents, the measured data can be used to 
calibrate the phase fractions with the cut-off response of the liquid. This simple method allows accurate detection of very small water hold-ups $(<5 \%)$ within the near horizontal oil extracting pipeline.

Method of Analysis: Multiphase liquids constitute of composite dielectric materials, where changing the percentage of any component modifies the effective dielectric constant of the mixture fluid and influences its scattering/cutoff behaviour. This principle is adopted here to determine the water hold-p (geometrical ratio of water height to pipe internal diameter) of a pipe filled with oil $\left(\varepsilon_{\mathrm{r}}=2.15\right)$ and saline-water $\left(\varepsilon_{\mathrm{r}}=81\right)$. The measurement unit used is illustrated in figure 1 , where the pipe is represented by a circular waveguide. The position and depth of the coaxial probe are important in exciting the waveguide in an optimum manner. For a homogenously loaded pipeline, cut-off response can be expressed in terms of pipeline diameter $(D)$ and Bessel function constant $(S)$ as,

$$
f_{c}=\frac{\pi D \sqrt{\varepsilon \cdot \mu}}{S}
$$

The two phase mixture within the pipe resembles to an in-homogenously loaded waveguide, where cut-off response ranges between equivalent modes of the guide resulting from individual loading of the dielectrics [4]. Thus, limiting values of the modal characteristics, such as $\mathrm{f}_{\mathrm{c}, \mathrm{TE} 11 \text { (oil only) }}=2.7386 \mathrm{GHz}$, calculated using equation (1) are used to govern the simulation process.

Results: A finite element solver is used to simulate the two-phase mixture within the pipe for various combinations of oil and water contents. The 
reflection responses $\left(S_{11}\right)$ of the pipe with water level ranging from $1 \mathrm{~mm}$ $(2.2 \%)$ to $4 \mathrm{~mm}(4.6 \%)$ are plotted in figure 2 . Note that increasing water level increases the effective dielectric constant of the two-phase mixture and consequently reduces the cut-off response of the pipeline. The conductivity of the pipe metal is observed to affect the cut-off response in an inverse manner, although it has little effect on the changes in $S_{11}$ response with changing oilwater ratio.

The measurement unit (figure 1) consisted of a probe feed circular waveguide segment, connected to the Network analyzer. The position, size, inset and alignment of the SMA probe are carefully selected to achieve best excitation. Reflections from the side terminations are minimized by selecting thick terminators with equivalent dielectric property. The volume of water/oil, needed to achieve a required water hold-up level, is carefully calculated to achieve accurate calibration. Figure 3 superimposes the simulated and experimental cut-off response with respect to the water hold-up level of the two-phase mixture within the pipe. Note that a maximum error of $1.5 \%$ occurs for high water level, whereas low water level errors remain less then $0.4 \%$.

Conclusion: A simple method and apparatus for determining the water content of crude oil in a pipeline is disclosed. Predicted calibration chart, which relates the water hold-up and the cut-off response of the two-phase mixture within the pipe, is experimentally verified. Accurate prediction of small $(<5 \%)$ water holdup in a horizontal or near horizontal oil carrying pipeline is demonstrated. 
Acknowledgment: The authors are grateful for the support of King Fahd University of Petroleum \& Minerals (KFUPM) and Schlumberger Dhahran Center for Carbonate Research, Dhahran, Saudi Arabia.

\section{References}

1 Michel Bérard and Ibrahim Babelli, System and Method for Measuring Flow in a Pipeline, WO 2006/072274, 13 July 2006.

2 Marioli D., Sardini E. and Taroni A., "Measurement of small Capacitance Variation", IEEE Trans. Instrumentation Meas., Vol 40, No. 2, pp. 426-428, 1991.

3 Wylie S.R., Shaw A. and Al-shamma's A.I., "RF sensor for multiphase flow measurement through an oil pipeline", Measurement Science and Technology, Vol. 17, pp. 2141-2149, 2006.

4 Baker-Jarvis J., Janezic M.D., Krupka J., "Broadband Dielectric Measurement of Liquids", Conference of Electrical Insulation and Dielectric Phenomena (CIEPD’04), 17-20 Oct. 2004, pp. 17-20, 2004.

\section{Authors' affiliations:}

Sheikh Sharif I., Alqurashi Khaled Y., Ragheb Hassan A. (Department of Electrical Engineering, King Fahd University of Petroleum \& Minerals (KFUPM), Dhahran-31261, Saudi Arabia)

Babelli I. (Schlumberger Dhahran Center for Carbonate Research, King Abdullah Science Park, Dhahran-31261, Saudi Arabia)

Corresponding author: Sheikh Sharif Iqbal; email: sheikhsi@kfupm.edu.sa

\section{Figure captions:}

Fig 1. (a) Cross section, (b) Side view of the measurement unit. (a=21.89mm, $\mathrm{b}=25.43 \mathrm{~mm}, l_{\mathrm{a}}=14.68 \mathrm{~mm}, l_{\mathrm{t}}=11.9 \mathrm{~mm}, d_{\mathrm{a}}=1.3 \mathrm{~mm}$ )

Fig 2. Reflection response of the two-phase (oil-water) mixture within the pipe, for four different level of water hold-up ( $1 \mathrm{~mm}$ to $4 \mathrm{~mm}$ ).

Fig 3. Measured and simulated response to calibrate water level against the cut-off property of the two-phase mixture within the pipe. For low water level, error between the experimental $(-)$ and predicted $(--)$ results are less than $0.4 \%$. 
Figure 1

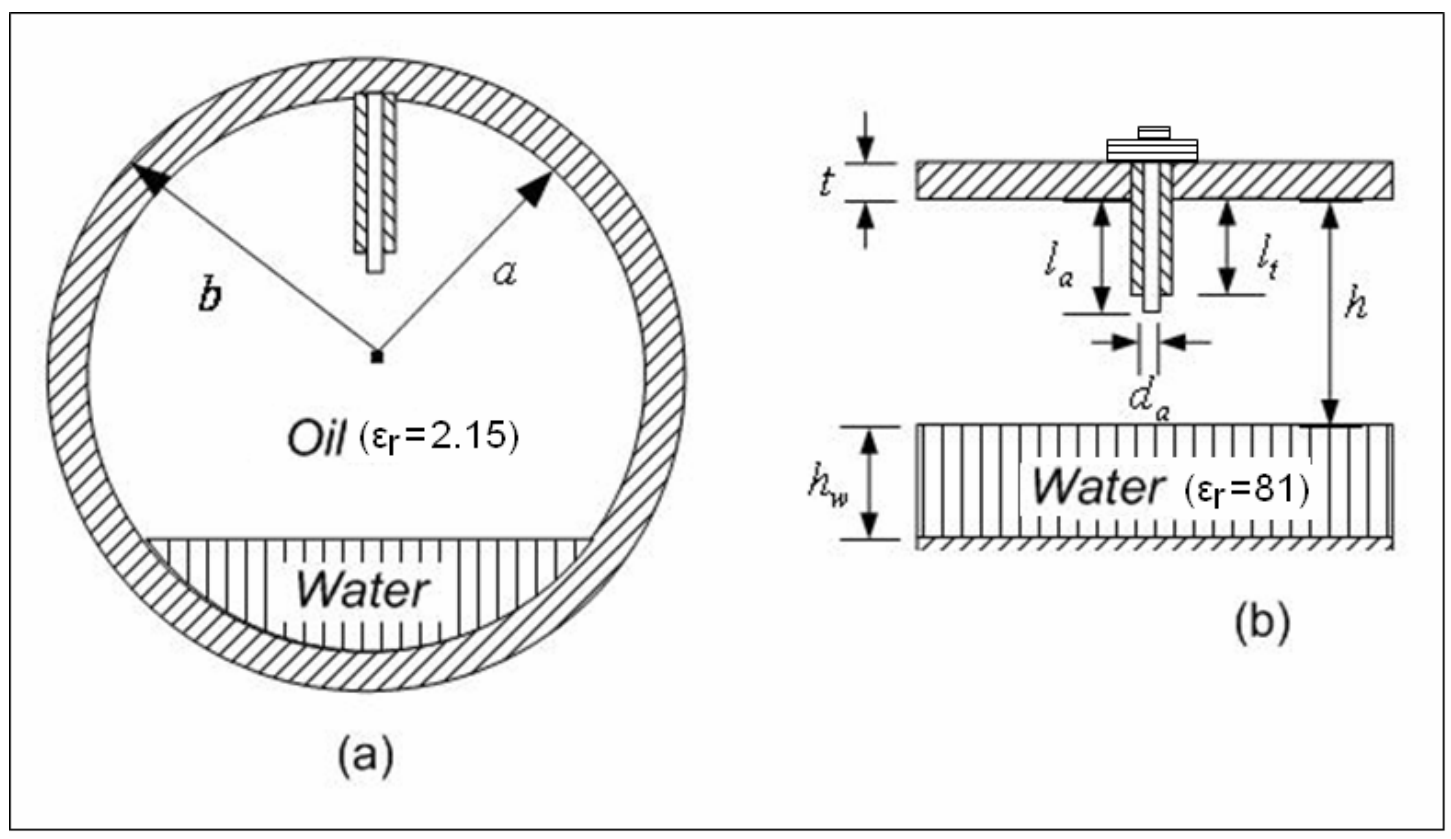


Figure 2

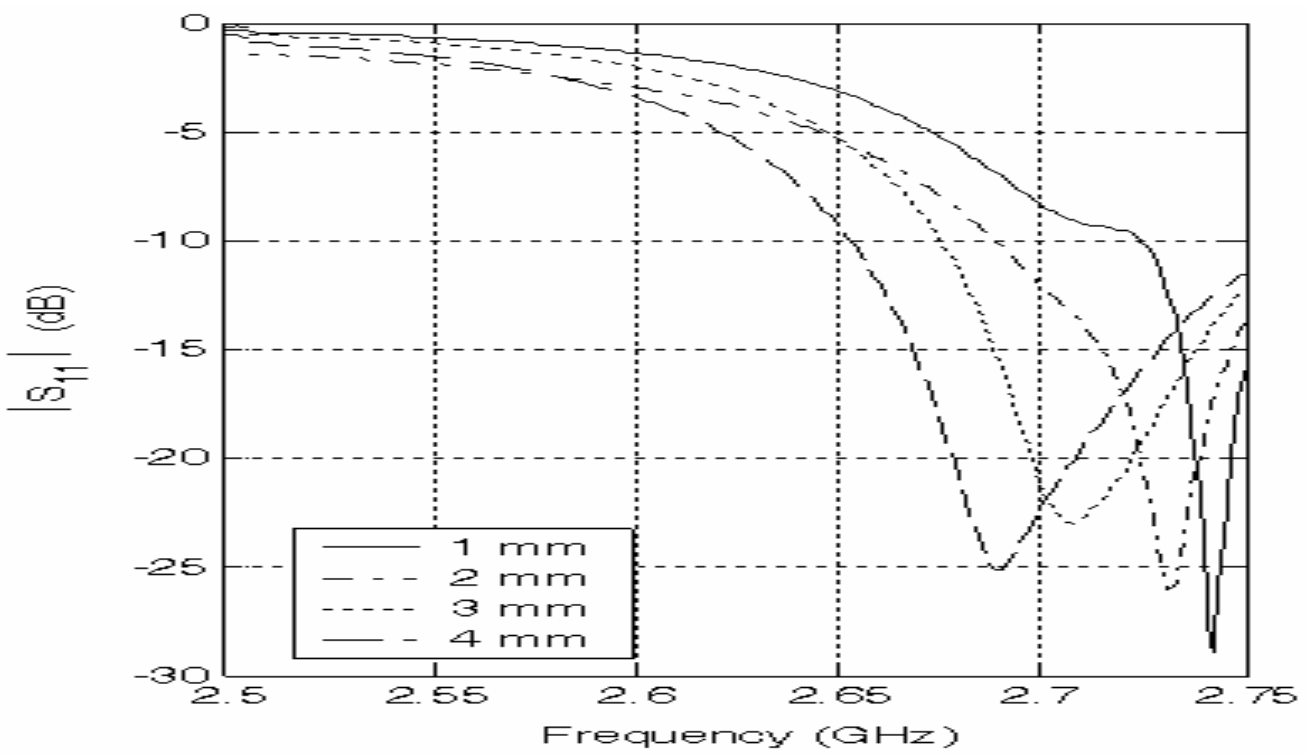


Figure 3

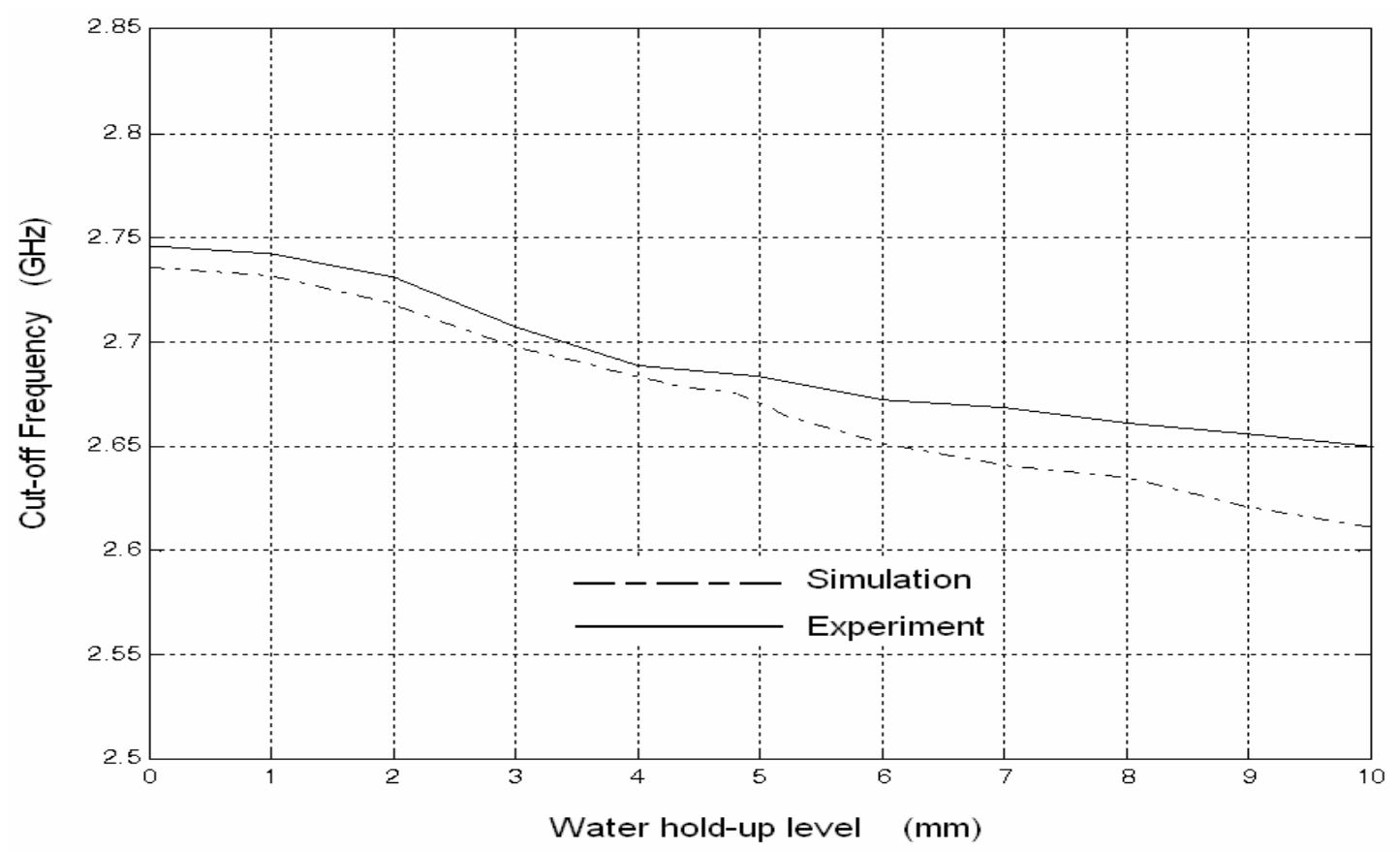

